\title{
Uso del modelo de aprendizaje invertido en un bachillerato público
}

\section{Using the flipped learning model in a public high school}

\author{
Waltraud Martínez-Olvera \\ Unidad de Educación Media Superior Tecnológica Industrial y de Servicios, Veracruz, México \\ miss.martinez.iex@hotmail.com \\ cetis134.dir@uemstis.sems.gob.mx \\ Ismael Esquivel-Gámez \\ Universidad Veracruzana, Veracruz, México \\ iesquivel@uv.mx
}

\begin{abstract}
Resumen
Los modelos tecno-educativos que se han venido desarrollando buscan la aplicación de las tecnologías de información y comunicación (TIC) más adecuada al diseño instruccional que se tiene planeado ejecutar. De entre los modelos revisados para el desarrollo del presente trabajo, el modelo de aprendizaje invertido presenta oportunidades de aplicabilidad en la educación pública de nivel medio superior. Dicho modelo propone, en términos generales, sustituir la cátedra del profesor por materiales de aprendizaje que puedan ser atendidos fuera de clase, mientras la típica tarea se convierte en actividades ejecutadas dentro del aula. A partir de ello, se describe una experiencia de invertir el aula en la materia "Comprensión de textos escritos en inglés como lengua extranjera" de quinto semestre de un bachillerato público en la ciudad de Xalapa, Veracruz (México). El apoyo tecnológico fue conformado por un curso, alojado en una plataforma Moodle, el cual maneja recursos digitales y actividades multimedia de práctica. Se eligió un enfoque cuantitativo con diseño cuasi-experimental de pre-prueba y post-prueba con grupo de control y experimental así como pruebas estandarizadas para la evaluación de la comprensión de textos. Los resultados muestran evidencias estadísticamente significativas que permiten realizar algunas consideraciones prometedoras.

Palabras clave

Modelo de aprendizaje invertido, inglés como lengua extranjera, alumnos de bachillerato público, comprensión escrita
\end{abstract}

\begin{abstract}
The purpose of the techno-pedagogical models that have been developed is to apply the most accurate information and communication technologies (ICT) use to the instructional design that is planned to be executed. Among the different models revised to develop the current research, the flipped learning model reveals application possibilities in the public high schools. In general, the flipped learning model proposes that the direct instruction is replaced by learning materials, which are delivered outside the class while typical homework activities are implemented in class time. On this basis, a flipped classroom experience was carried out in the "Reading comprehension in English as a foreign language" class in the fifth semester of a public high school in the city of Xalapa, Veracruz (Mexico). Digital resources and multimedia activities were developed and hosted in a Moodle platform. A quantitative-based approach was used along with a quasi-experimental design with control and experimental groups. Standardized tests for pre and post assessment of written text comprehension were also used. The results show statistically important evidences that allow to make some promising considerations.
\end{abstract}

\section{Keywords}

flipped learning model, English as a foreign language, high school students, reading comprehension 


\section{Introducción}

Uno de los retos, más ampliamente discutidos, para los profesionales de la educación en años recientes es el cambio de paradigma para lograr posicionar el proceso de aprendizaje como elemento central (Aguerrondo, 1999; Díaz-Barriga, 2003; Reigeluth, 2012; Tourón \& Santiago, 2015). En palabras de Prieto (2017), el reto es transitar de una enseñanza tradicional, enfocada en la transmisión de información por parte del docente, a una enseñanza que propicie espacios de discusión y actividades protagonizadas por el estudiante donde se priorice la interacción multidireccional alumno-alumno y docente-alumno en la construcción del conocimiento.

Desplazarse de un modelo de transmisión directa de la información a un modelo que privilegie la interacción y la evaluación formativa implica el diseño de escenarios que permitan transitar de espacios específicos de transmisión de conocimientos, protagonizados por el "sabio en la tarima" (Reigeluth, 2012), hacia la utilización de ambientes presenciales, virtuales y mixtos donde el estudiante sea quien dirija su avance en relación a las competencias desarrolladas en lugar de a un calendario de avance reglamentado (Reigeluth, 2012; Tourón \& Santiago, 2015).

Bajo la visión del aprendizaje centrado en el alumno, es necesario involucrar situaciones contextualizadas que aporten experiencias significativas (Díaz-Barriga, 2003). Como lo ha referido Prieto (2017), son varias las alternativas que paulatinamente se fueron desarrollando en línea con un enfoque activo de aprendizaje. Entre tales esfuerzos se cuenta la instrucción entre pares (peer instruction), la cual junto al método JiTT (just-intime teaching) prevé la instrucción fuera del aula y el intercambio constructivo de información tanto con los pares como con el profesor, durante la sesión presencial. Otros modelos activos incluyen el aprendizaje basado en equipos (team based learning) y la metodología semi-presencial o mixta.

Pese a que en numerosas aulas la realidad continúa siendo la enseñanza tradicional, se han propagado experiencias novedosas de intervención pedagógica que desde hace algunos años incluyen el uso de TIC. Si bien la introducción de herramientas tecnológicas ha acompañado la cátedra docente en sus diferentes etapas de evolución, el uso pedagógico de dichas herramientas es la clave para un adecuado proceso de enseñanza-aprendizaje. De manera que no solo se trata de agregar un instrumento, una herramienta o una estrategia nueva a la metodología tradicional, sino de implementar la didáctica apropiada.

Bajo la visión de la metodología mixta, que permite al estudiante autorregular el acercamiento a la información a través de materiales textuales impresos o multimedia, previo a la sesión virtual con el docente, se han desarrollado distintas experiencias educativas. Tal es el caso del denominado modelo de aprendizaje invertido, inicialmente conocido, en el contexto educativo mexicano, como aula inversa.

La extensa difusión del modelo de aprendizaje invertido, apadrinada por Aaron Sams y Jonathan Bergmann en 2012, ha generado un interés creciente alrededor del mundo,

Uso del modelo de aprendizaje invertido en un bachillerato público. Waltraud Martínez-Olvera, Ismael Esquivel-Gámez. . 
concretado en la Flipped Learning Global Initiative (FLGI), cuyo mayor promotor y evangelizador continúa siendo Bergmann (FLGI, 2016).

\section{Marco teórico-conceptual}

De manera general, el modelo de aprendizaje invertido consiste en voltear los roles y espacios tradicionales de enseñanza, de tal forma que el contenido conceptual típicamente impartido por el docente dentro del aula es atendido por el estudiante previo a las sesiones presenciales, mientras que la tarea caracterizada por ejercicios prácticos se retoma en clase. De este modo, se trata de movilizar la enseñanza directa hacia espacios individualizados de aprendizaje que, mediados por tecnología (audios, videos, plataformas), permitan capitalizar el aprendizaje del estudiante y liberar tiempo en el aula para implementar metodologías activas así como dar seguimiento y apoyo puntual a las necesidades que cada estudiante presente (Hamdan, McNight, McNight \& Arfstrom, 2013).

Para ubicar la base teórica del modelo de aprendizaje invertido se requiere tomar en cuenta aspectos de forma y fondo. Respecto a la forma, la revisión de los entornos mixtos de aprendizaje lo ubican como un sub-modelo de éste, conforme a la taxonomía de Staker y Horn (2012) y a la definición de Christensen, Horn y Staker (2010). En cuanto al fondo, hay que hacer referencia a la teoría constructivista de Vygotsky, el aprendizaje situado y, en la misma línea, a la Teoría del Aprendizaje Experiencial de Kolb (1984). En la figura 1, se encuentra una representación gráfica del sustento teórico referido.

La idea fundamental que subyace en la teoría socio-constructivista de Vygotsky señala que el funcionamiento cognitivo del individuo está ligado ("situado") a la interacción social y cultural dentro de contextos institucionales e históricos específicos. De manera que es necesario tomar en cuenta el entorno en el que ocurre la interacción de la comunidad de aprendizaje, las herramientas que median el proceso y las técnicas usadas para asistirlo (Bonk \& Cunningham, 1998).

En línea con el planteamiento anterior, el aprendizaje situado defiende la idea de que el saber teórico (el qué) es inseparable de la actividad que conlleve (el cómo). Se concibe al aprendizaje como un proceso activo, de construcción individual, que se facilita en relación directa con actividades desprendidas del contexto. Para ejemplificar cómo el aprendizaje es una conjugación inseparable, Brown, Collins y Duguid (1989) defienden que el uso de vocabulario variará de las definiciones formales referidas en un diccionario a las circunstancia de aplicación contextual, puesto que el factor cultural lo determina.

La teoría del aprendizaje experiencial tiene sus orígenes en los trabajos de Dewey, Lewis y Piaget. Se separa de las teorías cognitivas que enfatizan prioritariamente la adquisición, manipulación y posterior recuerdo de contenidos abstractos así como de las teorías más conductistas que tienden a negar la relevancia de la experiencia subjetiva en el aprendizaje (Kolb, 1984). Lejos de ser excluyente, Kolb ofrece una perspectiva integradora del aprendizaje que combine experiencia, percepción, conocimiento y conducta. De acuerdo al mismo autor, la teoría del aprendizaje experiencial es

Uso del modelo de aprendizaje invertido en un bachillerato público. Waltraud Martínez-Olvera, Ismael Esquivel-Gámez.. 
caracterizada por las principales aportaciones de los enfoques afines encontrados en Dewey, Lewis y Piaget, y sintetizados de la siguiente manera:

1) El aprendizaje es mejor concebido como un proceso que como un resultado.

2) El aprendizaje es un proceso continuo basado en experiencias, por lo que implica crear y recrear, no se reduce a adquirir o transmitir.

3) El aprendizaje es un proceso holístico de adaptación, transforma la experiencia tanto en forma objetiva como subjetiva.

4) El aprendizaje implica una transacción comunicativa entre el individuo y su entorno.

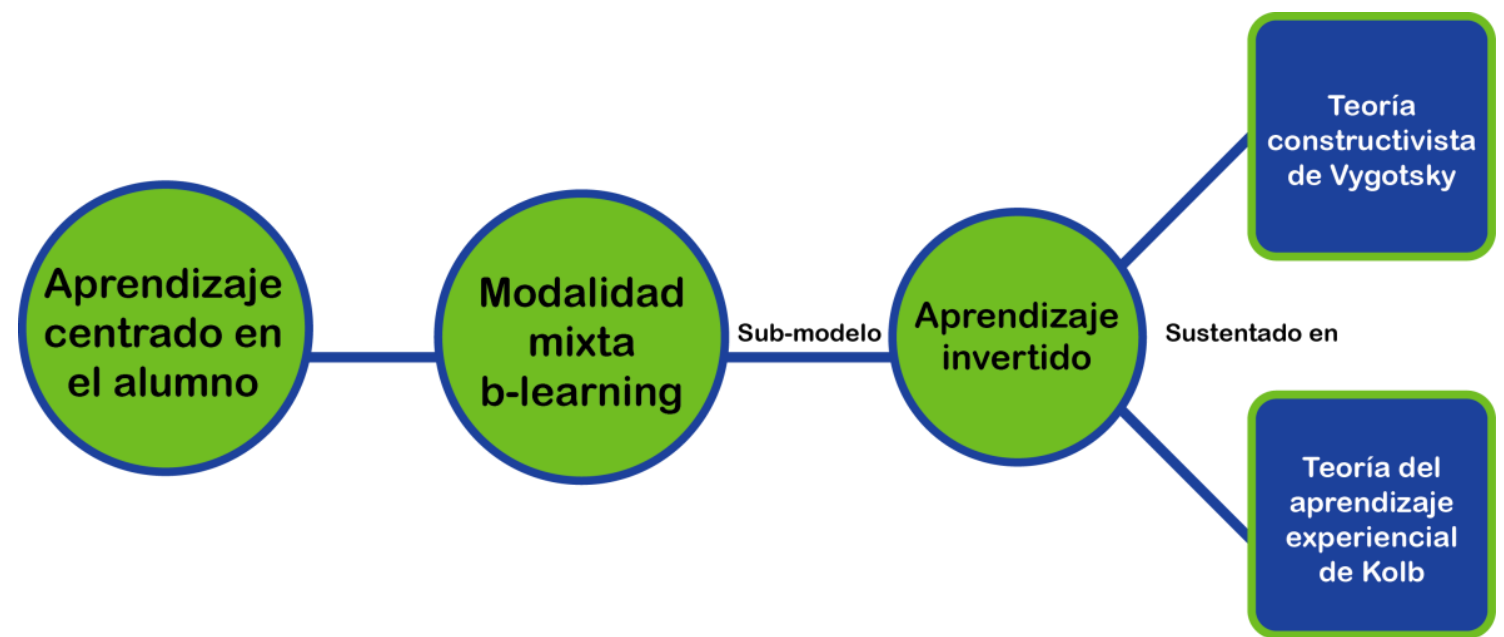

Figura 1. Sustento teórico del aprendizaje invertido. Fuente: Martínez-Olvera, EsquivelGámez y Martínez (2015a, p.165).

\section{El modelo del aprendizaje invertido en México}

La aceptación del modelo de aprendizaje invertido en Estados Unidos de Norteamérica propició el interés, por parte de las autoridades educativas en México, de implementarlo en las aulas; por lo que aprovechando la primera reunión nacional de academias de docentes en mayo 2014, se solicitó el uso del aula invertida en el nivel medio superior, estando a cargo de la comunicación el entonces Director General de Educación Tecnológica Industrial (DGETI), Ing. Alfonso Morán Moguel. De dicha reunión, los representantes de cada plantel asistente recogieron evidencias para trasladarlas a sus planteles de origen (Centro de Estudios Tecnológico industrial y de servicios 96 [CETis 96], 2014; Centro de Estudios Tecnológicos industrial y de servicios 134 [CETis 134] ${ }^{1}$, 2015). Sin embargo, su adopción en el sistema educativo se ha visto un tanto accidentada, siendo cuestionable su implementación en los planteles (Martínez-Olvera, Esquivel-Gámez \& Martínez, 2015a, 2015b).

Si bien se pueden encontrar comunidades en línea donde compartir experiencias de aplicación del modelo en el contexto latinoamericano, tal como el Flipped Learning Network-Latin America, aún son escasas las evidencias empíricas rigurosas (Hamdan, McNight, McNight \& Arfstrom, 2013; O'Flaherty \& Phillips, 2015; Yarbro, Arfstrom, McKnight \& McKnight, 2014). Al respecto, se han referido casos de éxito en el sistema educativo de apoyo particular (Observatorio de Innovación Educativa, 2014); sin

\footnotetext{
${ }^{1}$ Información recuperada mediante comunicado verbal en una reunión de docentes (3 de febrero, 2015).
}

Uso del modelo de aprendizaje invertido en un bachillerato público. Waltraud Martínez-Olvera, Ismael Esquivel-Gámez.. 
embargo, en sistemas de soporte público los intentos realizados refieren áreas de oportunidad (Madrid-García, 2016).

La escasez de pruebas concluyentes al respecto de la efectividad del modelo (MartínezOlvera, Esquivel-Gámez \& Martínez, 2015a; O'Flaherty \& Phillips, 2015) conllevan al análisis de las situaciones contextuales en las que se ha aplicado y una revisión profunda de los cuatro pilares y once indicadores que lo sustentan ${ }^{2}$. En este sentido, es que la FLGI promueve el denominado Flipped Learning 3.0 (FL 3.0), en donde se puntualizan los siguientes factores clave para la comprensión, difusión, adopción y actualización eficiente del modelo (FLGI, 2016):

- Reestructuración radical del rol del docente, del estudiante y del tiempo de interacción.

- Necesidad de un dominio profesional de la pedagogía y de las buenas prácticas docentes.

- Inversión de tiempo efectivo para la preparación del material y de los espacios académicos, lo cual requiere apoyo institucional.

- Selección del apoyo tecnológico más eficiente para el contexto, considerando el entrenamiento pertinente en uso.

- Fomento de procesos sencillos y relaciones productivas entre docente y discente.

A lo anterior, se incluye como prioridad la necesidad de tomar en cuenta el contexto y los conocimientos previos de los participantes a fin de organizar la experiencia pedagógica de forma más conveniente, haciendo énfasis en que el aprendizaje invertido no se limita al uso exclusivo de ciertas técnicas, sino que es una construcción y reorganización constante, siendo idóneo que el material de trabajo se encuentre accesible (Talbert, 2017).

\section{El presente estudio}

El objetivo del presente estudio fue evaluar los efectos de un modelo de instrucción bajo los principios del aprendizaje invertido en relación al desempeño lector en inglés de jóvenes bachilleres, provenientes de un centro escolar de sostenimiento público en México. La idea más difundida del modelo de aprendizaje invertido es que las explicaciones que proporciona el docente se trasladen fuera del aula mediante el uso de materiales de aprendizaje (videos, lecturas, audios). Sin embargo, experiencias informales previas sobre el comportamiento de los estudiantes de bachillerato con respecto a la consulta de material de trabajo (específicamente de tipo conceptual) fuera del aula no referían casos de éxito, como fue constatado durante el periodo julio-agosto 2015, en el curso taller de capacitación entre docentes del CETis 134.

Así pues, para llevar a cabo la propuesta, considerando la base filosófica del modelo de aprendizaje invertido, se analizaron los siguientes aspectos: 1) contexto; 2) nivel de comprensión lectora en inglés en la población objetivo; 3) accesibilidad al material en línea; y 4) las recomendaciones de Mackey y Ho (2008) sobre la percepción del rendimiento escolar que enseguida se explica.

\footnotetext{
${ }^{2}$ Para una revisión minuciosa de los mismos consultar Flipped Learning Network (2014). The four pillars of F-L-I-P. Retrieved from http://www.flippedlearning. org/definition.

Así como el análisis realizado en Martínez-Olvera, Esquivel-Gámez y Martínez (2015a, 2015b).
}

Uso del modelo de aprendizaje invertido en un bachillerato público. Waltraud Martínez-Olvera, Ismael Esquivel-Gámez.. 
Los estudios de Mackey y Ho (2008), reportan que cuando los alumnos trabajan con material en línea, la mejora de la percepción del rendimiento escolar tiene una asociación significativa con el acceso a los recursos dentro de la institución educativa, lo que no ocurre cuando el acceso es extra clase. Con lo anterior, se optó por abordar el material multimedia desde el aula presencial, todo lo cual requirió apoyo de la institución a fin de compaginar horarios de uso del aula de cómputo.

Como se ha mencionado, se aplicó el aula inversa para abordar los contenidos relacionados con la comprensión de textos en inglés, materia cuyo programa oficial estipula el acercamiento a la lectura académica mediante estrategias. Lo anterior precisa definir la comprensión de textos académicos en inglés y lo relativo a estrategias lectoras.

Comprensión de textos académicos en lengua extranjera. La comprensión de un texto es un proceso complejo que requiere la interacción texto-lector. La lectura, ya sea en lengua materna o lengua extranjera, parte de la decodificación del texto para extraer información, generar significado y conocimiento de nivel superior. Sin embargo, en lengua extranjera, la falta de exposición natural a la lengua meta, ocasiona carencias de vocabulario, estructuras gramaticales y, con ello, de fluidez (Grabe \& Stoller, 2002).

Tindall y Nisbet (2010) refieren que para una lectura propiamente comprensiva, se requiere decodificar con fluidez, tener un adecuado conocimiento previo y amplitud de vocabulario, así como utilizar apropiadamente estrategias de comprensión. Sin embargo, Valencia et al. (2010) sostienen que al elevarse el grado de estudios, la complejidad de la tarea lectora aumenta, ya que la lectura académica exige que los lectores ajusten su lectura al tipo de texto empleando estrategias para la comprensión como el detenerse y pensar o re-leer, por lo que la fluidez variará y no implicará un predictor en la comprensión. Ahora bien, para lectores de lengua extranjera, la decodificación está relacionada con el reconocimiento contextual de términos léxicos (Pasquarella, 2009), por lo que la amplitud de vocabulario (Eagleton \& Dobler, 2007; Grabe, 2009) así como la interacción entre el conocimiento disciplinar y la aplicación adecuada de estrategias permitirán descifrar con mayor precisión un texto (Ibáñez, 2007).

Estrategias de comprensión lectora. La enseñanza de la lectura en lengua extranjera requiere de una instrucción encaminada a mostrar explícitamente las acciones que se deben ejercer para abordar exitosamente un texto, incluyendo el cuándo, cómo y dónde aplicarlas (Anderson, 2003), reflejándose en acciones conscientes y auto-dirigidas, para que se puedan afianzar las siete habilidades lectoras recomendadas: lectura de exploración, lectura detallada, reconocimiento de significado contextual, comprensión de la estructura textual, lectura global, generación de inferencias y predicciones (Spratt, Pulverness y Williams, 2011). La enseñanza de estrategias en los tres momentos de lectura recomendados (antes, durante y después), permite la optimización del proceso (Aebersold \& Field, 1997).

La experiencia aquí referida fue guiada por el interés de implementar metodologías activas que estimularan el involucramiento de los jóvenes, considerando la idea de que la satisfacción en el proceso es parte esencial del éxito de una experiencia educativa. Así mismo, se tomó en cuenta que el conocimiento impartido en el contexto escolar no

Uso del modelo de aprendizaje invertido en un bachillerato público. Waltraud Martínez-Olvera, Ismael Esquivel-Gámez.. 
debe descontextualizarse de la influencia de las TIC en una sociedad globalizada (Finardi, Prebianca \& Schmitt, 2015). Lo anterior toma relevancia si lo que se pretende es estimular el desarrollo de la competencia comunicativa, afianzando la comprensión del texto escrito en inglés, lo cual se ha manifestado como una necesidad entre los estudiantes mexicanos (O’Donoghue, 2015). Con lo anterior se planteó como pregunta de investigación: ¿en qué grado un diseño instruccional, basado en los principios del aprendizaje invertido, impacta sobre el rendimiento en la comprensión de textos en inglés académico, en estudiantes de bachillerato público?

\section{Método}

Enfoque de la investigación. A fin de aportar datos sobre el efecto de la instrucción basada en el modelo del aprendizaje invertido, en contraste con el aula tradicional, se eligió un enfoque cuantitativo con diseño cuasi-experimental de pre-prueba y postprueba con grupo de control y experimental, auxiliándose de manera secundaria por una encuesta, cuyos resultados son integrados en la interpretación. Digno de comentarse es que por necesidades institucionales, el acceso a la población objetivo definió las características de los participantes, delimitando la experimentación hacia grupos no equivalentes y de carácter histórico (Cardona, 2003; Manterola \& Otzen, 2015) o de control externo (Esteban, 2014), en la cual se compara un grupo que recibe una intervención con otro que recibió otro tratamiento o ha sido tratado con anterioridad.

Participantes. Se realizó una selección por conveniencia, teniendo acceso a un centro educativo de nivel medio superior y de sostenimiento público en la ciudad de Xalapa, Veracruz (México). El plantel que permitió el acceso y desarrollo de la investigación recibe población proveniente de instituciones públicas de la periferia de una zona urbana así como de pequeñas localidades aledañas.

Como se ha referido previamente, los grupos participantes tuvieron características no equivalentes, especialmente en número y etapa de participación. Dos circunstancias particulares propiciaron las características de la muestra: 1) haber solicitado la participación voluntaria de los estudiantes del grupo experimental, y 2) la necesidad de incluir participantes que hubiesen cursado las experiencias educativas anteriores con el mismo profesor.

En la experiencia de aula inversa participaron 19 estudiantes de quinto semestre, ciclo escolar 2016, cuyas edades fluctuaron entre los 16 y 20 años, siendo mayoría los de 17; $68 \%$ de los mismos pertenecientes al género masculino y el $32 \%$ restante, al femenino. Los estudiantes habían participado previamente en experiencias educativas que requerían la consulta de material en línea fuera de la institución y durante el primer semestre habían utilizado equipos de cómputo para la clase denominada "Tecnologías de la Información y Comunicación". Sin embargo, no habían tenido oportunidad de utilizar herramientas TIC en el aula para una materia distinta.

El grupo de control, cuyos miembros recibieron instrucción con formato tradicional en lápiz y papel, estuvo formado por 33 estudiantes de quinto semestre inscritos en el ciclo escolar 2015, con edades entre 16 y 19 años, en un promedio de 17; con un $6 \%$ del género femenino y el $94 \%$ del masculino.

Uso del modelo de aprendizaje invertido en un bachillerato público. Waltraud Martínez-Olvera, Ismael Esquivel-Gámez.. 
El curso elegido para aplicar la secuencia de aprendizaje inverso fue el correspondiente al de comprensión de textos escritos en inglés como lengua extranjera, enfatizando que el centro educativo no cuenta con mediciones propias o estandarizadas para medir el nivel de competencia alcanzado por los alumnos inscritos, por lo que sólo se utiliza el promedio obtenido en la materia como criterio de logro. Cabe mencionar que, tanto en el grupo de control como en el experimental, se utilizaron los mismos contenidos tanto en gramática, vocabulario, aproximación estratégica a la lectura y textos de apoyo. La diferencia radicó en el formato de presentación de los mismos, así como en el proceso de acercamiento tutor-tutorado, al aplicarse un diseño de aula inversa en el grupo experimental.

Criterio de logro. Para identificar el progreso lector de los participantes, se tomó en cuenta el marco de referencia utilizado en el sistema de bachilleres, siendo éste el Marco Común Europeo de Referencia para las Lenguas (MCERL). Así mismo, conforme el programa académico del bachillerato, los estudiantes elegidos debían desempeñarse en el nivel lector B1 del MCERL (Aviña et al., 2013). Sin embargo, puesto que el centro educativo donde se realizó la intervención no realiza mediciones objetivas de avance logrado, se recurrió al uso de una prueba previa a la experiencia educativa, donde se constató que la mayoría de los participantes no alcanzaba el nivel mínimo A1. Tal aspecto determinó la selección de los grupos, puesto que para comparar la ejecución alcanzada, solo se seleccionaron para conformar el grupo de control, algunos que habían participado previamente con el mismo docente.

\section{Instrumentos de medición}

Se utilizó, previo al inicio de la experiencia, un instrumento de identificación de nivel en comprensión lectora del inglés (Penguin Readers Kit, Penguin Readers Teacher's Guide: Placement Test), el cual evalúa vocabulario y gramática e indica nivel de comprensión respecto al MCERL (Penguin Longman Publishing, 2001).

Además, se aplicó la prueba TOEFL ITP, sección tres, en las mediciones previa y posterior, tomando en cuenta los objetivos de la instrucción en los aprendizajes finales. Al tratarse de un curso de comprensión de lectura en inglés, se seleccionaron las preguntas correspondientes a los aprendizajes esperados contenidas en la prueba, la cual se caracteriza por ser una evaluación objetiva estandarizada, de la que se utilizaron cuatro textos y se desprendieron un total de catorce preguntas como meta a alcanzar.

Adicional a los datos de carácter cuantitativo, se aplicó un cuestionario corto de tipo abierto suministrado vía la mensajería instantánea de teléfonos móviles, a los participantes del grupo experimental, encaminado a rescatar las subjetividades del proceso vivido. De esta manera se logró obtener respuesta solo de 10 participantes, como se explica en la sección de resultados.

\section{Instrucción inversa}

Conforme los indicadores F2 y F3 del Flipped Learning Network, revisar el análisis del contexto y los conocimientos previos de los estudiantes es el primer paso para diseñar la experiencia de aula inversa (Flipped Learning Network [FLN], 2014). De manera que se

Uso del modelo de aprendizaje invertido en un bachillerato público. Waltraud Martínez-Olvera, Ismael Esquivel-Gámez.. 
procedió conforme dichas recomendaciones, bien delineadas en el Flipped Learning 3.0 (Talbert, 2017).

El curso de comprensión de textos en inglés fue estructurado en ocho semanas para los participantes del aula inversa. Cada semana se organizó en cuatro sesiones de 100 minutos cada una, haciendo un total de poco más de 53 horas. Por su parte, el curso tradicional, que debería contar con 90 horas semestrales, se distribuye en 5 horas semanales, resultando en 65 horas efectivas aproximadamente. Para la estructura del curso, se retomaron los objetivos clave del programa habitual en cuanto a acercar al alumnado al uso de estrategias de lectura que promuevan la comprensión del texto académico a nivel global y en cuanto a la identificación de: detalles textuales, de elementos inferidos o no textuales simples y del significado del vocabulario por contexto, ayudándose de la estructura gramatical pertinente. Debido a que se trabajó con estudiantes cuya experiencia con la lectura en inglés era muy escasa, se consideraron textos de apoyo sencillos, con contenido familiar rescatados de Wikipedia bajo licencia Creative Commons Attribution-ShareAlike $3.0^{3}$ (ver anexo).

Como primer pilar del modelo de aprendizaje inverso, y conforme el indicador F1 (FLN, 2014), se requiere de un ambiente flexible, por lo cual y para garantizar el acceso del material al ritmo individual de los participantes, se eligió la plataforma educativa Moodle 3.5 como herramienta que albergara secuencias por unidad de avance, tutoriales, lecturas y material multimedia para la ejercitación en línea así como ejercicios descargables en versión impresa. En el nivel medio superior se cuenta, de manera oficial, con la sugerencia de uso de la plataforma Edmodo, pero fue descartada porque su estructura de tipo red social dificulta la recuperación y/o actualización de material y no cuenta con registro de frecuencia de acceso ni de calificaciones, lo cual obstaculiza el seguimiento puntual de la ejecución individual, aspecto primordial en el aprendizaje invertido.

Dentro de la plataforma Moodle se alojaron seis unidades de trabajo en secuencia lineal. Cada unidad estaba caracterizada por un tema o concepto principal, acompañado de ejercicios prácticos e instrucciones de secuencia además de explicar el objetivo a alcanzar, todo ello en Español. El contenido conceptual se dosificó mediante tutoriales multimedia breves, a los que cada participante accedió a su propio ritmo y en los que se explicitó la estrategia de lectura a abordar con ejemplos de su uso. Los tutoriales fueron elaborados con PowerPoint y convertidos a archivos HTML, mediante la herramienta iSpring, incorporando audio con el software Audacity. Para clarificar el contenido, se anexaron archivos con ejemplos adicionales en formato PowerPoint, de manera que los estudiantes que lo requirieran pudiesen recurrir al recurso.

Los indicadores L1, L2, I1, I2, I3, contenidos en el segundo y tercer pilar del FLN (2014) versan sobre los aspectos a considerar bajo un enfoque centrado en el aprendizaje, así como respecto al andamiaje educativo. En línea con ello, se realizó la selección de material, dosificación e inclusión del mismo para distintos niveles de comprensión.

3

https://en.wikipedia.org/wiki/Wikipedia:Text_of_Creative_Commons_AttributionShareAlike_3.0_Unport ed_License

Uso del modelo de aprendizaje invertido en un bachillerato público. Waltraud Martínez-Olvera, Ismael Esquivel-Gámez.. 
Posterior a la visualización del contenido conceptual, para el contenido procedimental, se programó la práctica con ejercicios no interactivos en formato Word, que podían ser abiertos en alguna pestaña alterna del navegador o bien impresos. Una vez identificado el nivel de logro de los participantes, se agruparon conforme a su ejecución, de manera que pudieran socializar los resultados de los ejercicios impresos así como recibir apoyo y observaciones del docente. Conforme los participantes recibían retroalimentación y despejaban dudas, procedían a realizar, de manera individual, los ejercicios multimedia correspondientes a la unidad de estudio, los cuales se elaboraron con la herramienta JclicAuthor.

Las actividades generadas por la citada herramienta otorgan evaluación inmediata sobre la actividad realizada, permitiendo identificar lo aprendido o bien las áreas de oportunidad. Tal característica proporciona una herramienta esencial para el docente en aula inversa, ya que el cuarto pilar del modelo corresponde a la evaluación formativa, lo más cercana e individualizada posible (indicadores P1 y P2) (FLN, 2014). Así mismo, se promovió la consulta del glosario elaborado en la plataforma o cualquier otro diccionario en línea, de acuerdo a la preferencia de cada estudiante.

Al término de cada unidad se realizó una evaluación de tipo formativa. Los participantes tuvieron acceso a los recursos tanto dentro como fuera de la institución, por lo que pudieron abordar los materiales para repaso de aquellos temas en los que se observaba oportunidad de mejora.

Al finalizar las unidades programadas, se procedió a la aplicación del post-test y del cuestionario de opinión sobre el material, la estructura de la experiencia educativa y la percepción de lo aprendido.

\section{Resultados}

Prueba de identificación de nivel de lectura en inglés. La prueba se aplicó para corroborar el nivel de inglés conforme al MCERL. Pese a que el programa de estudios estipula un nivel B1 deseable, se encontró que sólo 10 participantes del total (grupo control y experimental), puntuaban en el mínimo A1. En cuanto a la puntuación de cada grupo, en el experimental, el 56\% de los participantes no alcanzó nivel alguno del MCERL, mientras que el $97 \%$ del grupo control tampoco lo hizo, evidenciando un nivel precario de manejo del inglés tanto en gramática como en vocabulario.

Sobre la comprensión lectora. El test TOEFL fue procesado mediante pruebas $\mathrm{T}$ para muestras relacionadas y los resultados indican ganancias tanto en el grupo experimental $(\mathrm{p}=0.000)$ como en el grupo de control $(\mathrm{p}=0.036)$. Ello implica que la implementación de estrategias de lectura para textos en inglés surte los efectos deseables sobre la comprensión del texto escrito. La evidencia aquí presentada se vuelve relevante al dimensionar que la población participante contaba con un bajo nivel de manejo del idioma, como ha sido señalado en el párrafo inmediato anterior.

Aunado a lo ya expuesto, para el grupo experimental, se corrobora la correlación de nivel medio entre el nivel de comprensión lectora y el promedio de calificación obtenido en las actividades multimedia $(r=0.523, p=0.026)$. Es decir, la herramienta

Uso del modelo de aprendizaje invertido en un bachillerato público. Waltraud Martínez-Olvera, Ismael Esquivel-Gámez.. 
tecnológica permitió monitorear la ejecución de los participantes arrojando una calificación promedio personalizada. Tal cualidad facilitó verificar fortalezas y debilidades en el avance de los participantes, así como retomar la aclaración de los temas no logrados. Para ello se tuvo a disposición los tutoriales, el material de apoyo adicional y la asesoría del profesor. Verificar la correlación entre la ejecución promedio en las actividades multimedia y los resultados en comprensión lectora permite conjeturar que el uso de los recursos aportó beneficios al proceso final.

Al comparar la ejecución entre el grupo de control y el experimental, se encontró una diferencia estadísticamente significativa entre los puntajes de lectura comprensiva, luego del tratamiento. Conforme a los resultados obtenidos de la prueba $\mathrm{T}$ para muestras independientes $(\mathrm{t}=-5.408, \mathrm{p}=0.000)$, se encontró un incremento significativo en las medias, a favor del experimental (12.17 vs. 6.52). Si bien los resultados globales permiten corroborar la influencia positiva del curso sobre los niveles de comprensión lectora en inglés, la comparación de las variantes del mismo curso hace posible determinar la ventaja del formato de aula inversa diseñado exprofeso por sobre la clase tradicional.

Otros factores asociados a diferencias del grupo experimental. A fin de verificar la correlación existente entre factores adicionales a la ejercitación con actividades multimedia y que pudieran influir en los niveles de comprensión lectora, antes y después del tratamiento, se examinaron diversos niveles de asociación que enseguida se abordan.

En primer lugar, se buscó cotejar la relación entre la comprensión lectora y elementos previos a la intervención. Enseguida, se realizó el procedimiento estadístico correspondiente para verificar asociación entre los resultados de TOEFL y la prueba Penguin Readers. El puntaje de ubicación de nivel inicial mediante la prueba Penguin Readers, no arrojó correlación alguna significativa para ambos grupos. Tales resultados refieren que los cambios en comprensión lectora pueden atribuirse al tratamiento.

Adicionalmente, se examinó la posible relación entre los puntajes de comprensión lectora y la frecuencia de uso de los materiales multimedia de lo cual se detectó una correlación baja y no significativa $(\mathrm{r}=0.181, \mathrm{p}=0.472)$. En cuanto al nivel de asociación con la frecuencia total de uso de los recursos multimedia (glosarios, actividades y tutoriales), no se muestra relación alguna en los resultados globales. Es un dato paradójico no haber encontrado una correlación significativa entre la frecuencia de uso de los recursos y la ejecución final, aunque sí la hay entre esta última y el promedio logrado en las actividades multimedia, lo que conduce a recomendar pruebas subsecuentes a fin de obtener resultados definitivos.

Sobre la opinión de los participantes. La encuesta aplicada a los participantes voluntarios del grupo experimental, constó de cuestionamientos de tipo abierto, en la que se ejecutó un análisis de contenido temático, preservando fundamentalmente la identificación del contenido explícito (Andréu, 2001). La encuesta se envió al total de participantes en la experiencia de aula invertida, sin embargo sólo se recabaron 10 respuestas. Al respecto, pudo haber influido que la encuesta se aplicara en periodo de exámenes y especificando respuesta voluntaria.

Uso del modelo de aprendizaje invertido en un bachillerato público. Waltraud Martínez-Olvera, Ismael Esquivel-Gámez.. 
Conforme a lo anterior, se solicitó a los participantes que compararan la clase en aula inversa con el método tradicional en tres dimensiones: material utilizado, evaluaciones y aprendizaje, recabando los datos que a continuación se presentan. En cuanto al material utilizado en el aula inversa, con respecto al aula habitual, el 50\% de las respuestas se orientaron a mencionar mayor facilidad en el uso de la plataforma que con los textos. El $100 \%$ de los encuestados aludió que el material fue mejor que el utilizado en el aula regular, calificándolo como: "dinámico", "no te aburre", "muy digerible", "cómodo y flexible", "práctico" y "más fácil". A la comparación del aprendizaje percibido, el $60 \%$ de las respuestas señalaron que la forma en que se desarrolló la clase en aula inversa les parecía mejor. Con respecto a las diferencias entre la forma de calificar, $90 \%$ de los encuestados nuevamente recurrió al término "mejor" a favor del aula inversa; 60\% apuntó que la evaluación se otorga a tu "conocimiento", especificando que en aula tradicional se pueden copiar los resultados obtenidos por otros participantes. Si bien las respuestas otorgadas por los respondientes apuntaron en sentido positivo, se debe tomar en cuenta que nueve de los participantes no respondieron la encuesta. Por lo tanto, se debe ser cauto con la interpretación.

\section{Conclusiones}

Es un hecho que los cambios sociales, políticos y tecnológicos implican una reestructuración seria del sistema educativo tradicional, a pesar de los esfuerzos reformistas que se han implementado. En este sentido, se ha presenciado la intención de cambio enfocada en la adición de apoyos didácticos al proceso educativo pero sin un modificación real en el modelo de fondo. Así pues, antes que tecnología para mediar el proceso educativo, es necesario analizar lo que las ciencias de la educación aportan a la metodología y a las técnicas de la enseñanza. Es en tal escenario, que surgen nuevos modelos educativos, que mediados por tecnología, pretenden priorizar el proceso de aprendizaje, enfatizando las particularidades de cada estudiante, como lo propone el modelo de aprendizaje invertido.

Si bien los casos de éxito en países extranjeros pudieran tomarse como referente para adoptar la propuesta de aula inversa, es recomendable realizar los ajustes pertinentes a cada contexto y contar con evidencia que respalde su idoneidad. Ello motivó el planteamiento e investigación sobre los efectos de un modelo de instrucción de aula inversa en el desempeño lector en inglés de jóvenes bachilleres, de una escuela pública. De este modo se pudieron reunir evidencias estadísticamente significativas, que permiten realizar algunas consideraciones prometedoras, como enseguida se expone.

En primer lugar, se constata que el grupo participante en la experiencia de aula inversa alcanzó una mejor ejecución en lectura en contraste con el grupo que participó en el aula regular, pese a que los contenidos fueron los mismos. Esto hace suponer que la exposición al material conceptual bajo un formato más individualizado puede reflejar mayores ganancias a la hora de aprehenderlo. Tal apreciación se apoya en los resultados que muestran un nivel de asociación significativo entre los puntajes de comprensión y el promedio de calificación obtenido en las actividades multimedia. Sin embargo, debido a las características no equivalentes de los grupos, se requieren subsecuentes pruebas que aporten resultados definitivos.

Uso del modelo de aprendizaje invertido en un bachillerato público. Waltraud Martínez-Olvera, Ismael Esquivel-Gámez.. 
En segundo lugar, si bien la tecnología no es la respuesta para la optimización del proceso de enseñanza, el uso de tutoriales con acceso individual parece haber simplificado el acceso a los temas de estudio y su ejercitación, lo cual, aunado al agrupamiento de los participantes por nivel de ejecución, permitió otorgarles asesorías correspondientes a sus necesidades. Es decir, la herramienta tecnológica aunada a la presencia docente, en este caso, funcionó para extender la asesoría del profesor.

Cabe recordar que los grupos comparados participaron con el mismo docente e interactuaron con materiales similares, excepto por la presentación de los mismos (impreso vs. digital). En experiencias informales previas, se había observado la renuencia de los alumnos para realizar la revisión de los contenidos conceptuales fuera del aula. La presente investigación se propuso solventar tales inconvenientes siguiendo las recomendaciones de Mackey y Ho (2008) sobre el uso de los recursos dentro del plantel educativo. El formato elegido parece haber aportado beneficios respecto a la participación y la percepción de mejora de los alumnos, en línea con lo que refieren los autores citados.

En tercer lugar, la referencia que hacen los participantes hacia la forma de evaluar que vivieron en la experiencia implementada, en contraposición con la tradicional, provoca la reflexión respecto al componente formativo que debe acompañar el proceso de aprendizaje. La evaluación formativa es útil siempre que se realice en el momento preciso y de manera contingente. En el presente caso, la evaluación inmediata y automatizada de las actividades realizadas así como las observaciones del docente a la ejecución de los participantes, parece haber surtido un efecto más satisfactorio en los estudiantes.

Finalmente, la disposición del modelo de aula inversa dentro de la institución educativa, ha permitido el acceso al contenido, el avance individual, el registro certero de cada participante, así como la aclaración de dudas surgidas en el momento oportuno.

La instrucción girada por las autoridades educativas mexicanas respecto a la implementación del modelo de aula invertida en el bachillerato, trajo consigo posturas discordantes. Por un lado, docentes renuentes a participar de un método desconocido; por otro, directivos que resolvieron el cumplimiento de las disposiciones solicitando a su personal agregar en la planeación el uso de algún recurso audiovisual en línea. Con tales acciones no se obtienen directrices claras que respalden el funcionamiento del modelo. En cambio, la aportación de la presente experiencia permite vislumbrar la posibilidad de aplicar el modelo de aula inversa en los bachilleratos de sostenimiento público. Lo cual es viable siempre que se respete como principio clave, en coincidencia con las reflexiones de Tourón y Santiago (2015), un proceso formativo que considere la tecnología idónea al contexto y el diseño pedagógico que promueva la labor del estudiante respetando sus singularidades.

Presentación del Artículo: 14 de mayo de 2017 Fecha de aprobación: 24 de septiembre de 2018 Fecha de publicación: 31 de octubre de 2018 
Martínez-Olvera, W. y Esquivel-Gámez, I. (2018). Uso del modelo de aprendizaje invertido en un bachillerato público. RED Revista de Educación a Distancia, 58. Consultado el (dd/mm/aaaa) en http://www.um.es/ead/red/58

\section{Financiación}

Esta investigación no ha recibido ninguna subvención específica de los organismos de financiación en los sectores públicos, comerciales o sin fines de lucro.

\section{Referencias}

Aebersold, J., \& Field, M. (1997). From reader to reading teaching: Issues and strategies for second language classrooms. Cambridge: Cambridge University Press.

Aguerrondo, I. (1999). El nuevo paradigma de la educación para el siglo. Recuperado del sitio de Internet Organización de Estados Iberoamericanos para la Educación, la Ciencia la http://www.oei.es/historico/administracion/aguerrondo.htm

Anderson, N. (2003). Scrolling, clicking, and reading English: online reading strategies in a second/foreign language. Reading Matrix: An International Online Journal, 3(3), 1-33. Recuperado de http://www.readingmatrix.com/articles/anderson/article.pdf

Andréu, J. (2001). Las técnicas de análisis de contenido: una revisión actualizada. Documento de trabajo, S2001/03, Centro de Estudios Andaluces. Recuperado de http://public.centrodeestudiosandaluces.es/pdfs/S200103.pdf

Aviña, C., Canizales, G., Corrales, L., Galindo, P., Meillón, F., Mendoza, H. Ornelas, L. (2013). Programa de estudios de inglés. Bachillerato tecnológico. Secretaria de Educación Pública. Subsecretaría de Enseñanza Media Superior: México.

Bonk, C. J., \& Cunningham, D. J. (1998). Searching for learner-centered, constructivist, and sociocultural components of collaborative educational learning tools. En C. J. Bonk \& K. S. Kim (Eds.), Electronic collaborators: learner-centered technologies for literacy, apprenticeship, and discourse (pp. 25-50). New Jersey: Erlbaum.

Brown, J., Collins, A., \& Duguid, P. (1989). Situated cognition and the culture of learning. Educational researcher, 18(1), 32-42.

Cardona, A. (2003). Diseños cuasiexperimentales. Facultad Nacional de Salud Pública. Universidad de Antioquia. Recuperado de http://www.sld.cu/galerias/pdf/sitios/renacip/disenos_cuasiexperimentales.pdf

Centro de Estudios Tecnológico industrial y de servicios 96 (Productor) (2014). Informe reunión nacional de academias de la Dirección General de Educación 
Tecnológica Industrial [video en línea]. Recuperado de https://www.youtube.com/watch?v=QPY0dsf9bt4

Christensen, C., Horn, M., \& Staker, H. (2013). Is K-12 blended learning disruptive: An introduction of the theory of hybrids. Recuperado del sitio de Internet del Clayton Christensen Institute: http://www.christenseninstitute.org/wpcontent/uploads/2013/05/Is-K-12-Blended-Learning-Disruptive.pdf

Díaz-Barriga, A. (2003). Cognición situada y estrategias para el aprendizaje significativo. Revista electrónica de investigación educativa, 5(2), 1-13.

Eagleton, M., \& Dobler, E. (2007). Reading the Web: strategies for internet inquiry solving problems in the teaching of literacy. New York: Guilford Press.

Esteban, M. (2014). Diseños experimentales en salud pública: principales características de los modelos, técnicas de análisis y casos. Research, 1(597). doi: http://dx.doi.org/10.13070/rs.es.1.597

Finardi, K., Prebianca, G., \& Schmitt, J. (2015). English distance learning: possibilities and limitations of MEO for the Flipped Classroom. Revista Brasileira de Linguística Aplicada, 16(2), 181-208.

Flipped Learning Global Iniciative (2016). Flipped learning core principles. Recuperado de http://flglobal.org/

Flipped Learning Network [FLN] (2014). The four pillars of F-L-I-P. Recuperado de http://www.flippedlearning. org/definition

Grabe W., \& Stoller, F. (2002). Teaching and researching reading. New York: Routledge.

Grabe, W. (2009). Reading in a Second Language: Moving to Theory from Practice. New York: Cambridge University Press.

Hamdan, N., McNight, P., McNight, K., \& Arfstrom, K. (2013). A review of flipped learning. USA: Flipped Learning Network, Pearson and George Mason University.

Ibáñez, R. (2007). Comprensión de textos disciplinares escritos en inglés. RLA. Revista de lingüística teórica y aplicada, 45(1), 67-85. Recuperado de http://www.scielo.cl/scielo.php?script=sci_arttext\&pid=S071848832007000100005

Kolb, D. A. (1984). Experiential learning: experience as the source of learning and development. Englewood Cliffs, NJ: Prentice Hall.

Mackey, T. P., \& Ho, J. (2008). Exploring the relationships between Web usability and students' perceived learning in Web-based multimedia (WBMM) tutorials. Computers \& Education, 50(1), 386-409. 
Madrid-García, E. (noviembre, 2016). Propuesta de Aula Invertida en cursos propedéuticos de bachillerato. Trabajo presentado en el II Coloquio Interinstitucional BUAP-ITSON-UV de Investigación e Innovación en Sistemas y Ambientes Educativos CIIISAE-2016, Boca del Río, Veracruz.

Manterola, C., \& Otzen, T. (2015). Estudios experimentales 2a parte: estudios cuasiexperimentales. International Journal of Morphology, 33(1), 382-387.

Martínez-Olvera, W., Esquivel-Gámez, I., \& Martínez, J. (2015b). Aula invertida o modelo invertido de aprendizaje: origen, sustento e implicaciones. En I., Esquivel-Gámez (Coord.), Los modelos Tecno-Educativos, revolucionando el aprendizaje del siglo XXI (pp. 137-154). Recuperado de shorturl.at/bvxEW

Martínez-Olvera, W., Esquivel-Gámez, I., \& Martínez, J. (2015a). Acercamiento teórico-práctico al modelo de aprendizaje invertido. En M. Osorio \& I. E. Peón (Eds.), Alternativas para nuevas prácticas educativas (pp. 158-172). México: Amapsi Editorial.

O’Donoghue, J. (Coord.). (2015). Sorry. El aprendizaje del inglés en México. Recuperado del sitio de Internet de Mexicanos Primero: http://www.mexicanosprimero.org/index.php/educacion-en-mexico/como-estala-educacion/estado-de-la-educacion-en-mexico/sorry-2015

Observatorio de Innovación Educativa (octubre, 2014). Aprendizaje invertido. Edu Trends, Tecnológico de Monterrey, 1-29. Recuperado de http://www.sitios.itesm.mx/webtools/Zs2Ps/roie/octubre14.pdf

O'Flaherty, J., \& Phillips, C. (2015). The use of flipped classrooms in higher education: A scoping review. The Internet and Higher Education, 25, 85-95.

Pasquarella, A. (2009). Reading comprehension in adolescent first and second language learners: A comparison of simple and multi-component models. (Tesis de maestría). Recuperada de ProQuest Dissertations and Theses. (Order No. MR54241).

Penguin Longman Publishing (2001). Penguin Readers Kit. Penguin Readers Teacher's Guide: Placement Test. London: Pearson Education.

Prieto, A. (2017). Flipped learning: aplicar el modelo de aprendizaje inverso. Madrid: Narcea Ediciones.

Reigeluth, C. (2012). Instructional Theory and Technology for the New Paradigm of Education. RED, Revista de Educación a Distancia. Recuperado de http://revistas.um.es/red/article/view/270781

Spratt, M., Pulverness, A., \& Williams, M. (2011). The TKT Course Modules 1, 2 and 3. Cambridge: Cambridge University Press.

Uso del modelo de aprendizaje invertido en un bachillerato público. Waltraud Martínez-Olvera, Ismael Esquivel-Gámez.. 
Staker, H., \& Horn, M. (2012). Classifying K-12 Blended Learning. Innosight Institute.

Talbert, R. (8 de marzo de 2017). Entrevista de Jon Bergmann en BAM Radio Network [audio]. Flipped Learning 3.0: The Paradigm Shift That Changes Everything. Recuperado de http://www.bamradionetwork.com/the-flip-side-with-jonbergmann/4286-flipped-learning-3-0-the-one-idea-that-changes-everything

Tindall, E., \& Nisbet, D. (2010). Exploring the Essential Components of Reading. Journal of Adult Education, 39(1), 1-9. Recuperado de http://files.eric.ed.gov/fulltext/EJ891080.pdf

Tourón, J., \& Santiago, R. (2015). El modelo Flipped Learning y el desarrollo del talento en la escuela. Revista de Educación, 368, 196-231.

Valencia, S., Smith, A., Reece, A., Li, M., Wixson, K., \& Newman, H. (2010). Oral reading fluency assessment: Issues of construct, criterion, and consequential validity. Reading Research Quarterly, 45(3), 270-291. doi: http://dx.doi.org/10.1598/RRQ.45.3.1

Yarbro, J., Arfstrom, K. M., McKnight, K. \& McKnight, P. (2014). Extension of a Review of Flipped Learning. Flipped Learning Network. Recuperado de http://flippedlearning.org/cms/lib07/VA01923112/Centricity/Domain/41/Extensi on\%20of\%20FLipped\%20Learning\%20LIt\%20Review\%20June\%202014.pdf

Anexo. Ejemplo de texto utilizado

Jules Gabriel Verne (8 February 1828 - 24 March 1905) was a French novelist, poet, and playwright best known for his adventure novels and his profound influence on the literary genre of science fiction.

Verne was born to bourgeois parents in the seaport of Nantes, where he was trained to follow in his father's footsteps as a lawyer, but quit the profession early in life to write for magazines and the stage. His collaboration with the publisher Pierre-Jules Hetzel led to the creation of the Voyages extraordinaires, a widely popular series of scrupulously researched adventure novels including Journey to the Center of the Earth (1864), Twenty Thousand Leagues Under the Sea (1870), and Around the World in Eighty Days (1873).

Verne is generally considered a major literary author in France and most of Europe, where he has had a wide influence on the literary avant-garde and on surrealism. His reputation is markedly different in Anglophone regions, where he has often been labeled a writer of genre fiction or children's books, largely because of the highly abridged and altered translations in which his novels are often reprinted. 